\title{
Estimation of Stator Resistance and Rotor Flux Linkage in SPMSM Using CLPSO with Opposition-Based-Learning Strategy
}

\author{
Jian He and Zhao-Hua Liu \\ School of Information and Electrical Engineering, Hunan University of Science and Technology, Xiangtan 411201, China \\ Correspondence should be addressed to Zhao-Hua Liu; zhaohualiu2009@hotmail.com
}

Received 31 March 2016; Revised 26 May 2016; Accepted 27 June 2016

Academic Editor: Qiao Zhang

Copyright ( 2016 J. He and Z.-H. Liu. This is an open access article distributed under the Creative Commons Attribution License, which permits unrestricted use, distribution, and reproduction in any medium, provided the original work is properly cited.

\begin{abstract}
Electromagnetic parameters are important for controller design and condition monitoring of permanent magnet synchronous machine (PMSM) system. In this paper, an improved comprehensive learning particle swarm optimization (CLPSO) with opposition-based-learning (OBL) strategy is proposed for estimating stator resistance and rotor flux linkage in surface-mounted PMSM; the proposed method is referred to as CLPSO-OBL. In the CLPSO-OBL framework, an opposition-learning strategy is used for best particles reinforcement learning to improve the dynamic performance and global convergence ability of the CLPSO. The proposed parameter optimization not only retains the advantages of diversity in the CLPSO but also has inherited global exploration capability of the OBL. Then, the proposed method is applied to estimate the stator resistance and rotor flux linkage of surfacemounted PMSM. The experimental results show that the CLPSO-OBL has better performance in estimating winding resistance and PM flux compared to the existing peer PSOs. Furthermore, the proposed parameter estimation model and optimization method are simple and with good accuracy, fast convergence, and easy digital implementation.
\end{abstract}

\section{Introduction}

In recent years, permanent magnet synchronous machines (PMSM) have been widely applied in industrial servo control system and renewable energy power generation system [1$3]$, as they possess superiority in high power density, torque response, high efficiency performances, and so forth. It is necessary to exactly obtain the parameters of PMSM for assisting controller design, speed regulation, and condition monitoring in reality industrial drive system [4]. Particularly, the most important physical parameters such as the stator resistance and the rotor PM flux linkage are the indicators of system health status. For example, the stator resistance can be seen as the indicator of stator temperature due to metal thermal efficiency, since the machine service life will be damaged if the temperature exceeds its critical range. The demagnetization in PM will influence machine electromagnetic torque output performance [5]. However, the PMSM is a typical nonlinear time-varying dynamic system, whose physical parameters are easily sensitive to the changes of environment such as noise and temperature. Thus, technologies for estimating the winding resistance and rotor flux linkage of
PMSM have become an important task for machine control [6]. Existing literatures mainly focus on online estimation and algorithms including extended Kalman filter (EKF) $[6,7]$, model reference adaptive system (MRAS) $[8,9]$, recursive least-square (RLS) methods [10, 11], and neural network (NN) [12] are widely employed. However, the aforementioned methods possess some drawbacks such as error convergence, unsteadiness, and high computing expense in the process of PMSM estimation.

Recently, inspired by biological computing, some researchers try to use evolutionary computation techniques to estimate the parameters of PMSM as the evolutionary algorithms have the ability to obtain a suitable set of parameter values via optimizing objective function between the system model and the actual ones. The particle swarm optimization (PSO) has recently been introduced as an attractive optimization technique in system identification and successfully applied in PMSM parameter estimation [2, 5, 13-15], due to its simple implementation, little control parameters, multidirectional search, and fast convergence. Since the basic PSO easily gets trapped in local minima when solving the complex nonlinear problem of PMSM parameter 
identification, some enhanced operators are introduced into the PSO and produced new hybrid PSO to design the parameters estimator of PMSM. For example, a coevolution based parameter estimator by combining multiple population PSO and artificial immune algorithm was investigated to improve multiparameter estimation performance of PMSM [2]. In order to improve the efficiency of parameter identification, a parallel implementation using an immunecooperative dynamic learning particle swarm optimization (PSO) algorithm with multicore computation architectures is presented for PMSM parameter estimations [5]; another method of graphic processing unit (GPU) accelerated parallel coevolutionary immune PSO was designed for parameter estimation and temperature monitoring of a PMSM [15], for which the performance of the parameter estimation was significantly improved by those new PSO methods.

In this paper, in order to estimate the stator resistance and rotor flux linkage of surface-mounted PMSM effectively, the parameter estimation of PMSM is converted to an optimization problem and then a novel comprehensive learning particle swarm optimization (CLPSO) with oppositionlearning strategy is proposed to explore optimal parameter, the proposed parameter optimization method called CLPSOOBL. The CLPSO was firstly proposed by Liang et al. [16], where all the flying directions of individuals are updated by randomly selected particle of the whole population during the iteration process and it is superior in diversity kept for solving multimodal optimization problem. In order to improve the global convergence of the CLPSO, an oppositionbased-learning (OBL) strategy is used for Pbest particles learning and helps it jump out of local optima. OBL is a reinforcement learning strategy using computing and counter computing simultaneously, which can be used to accelerate the convergence performance of other evolutionary algorithms [17]. Finally, the proposed CLPSO-OBL is applied to estimate the stator resistance and rotor flux linkage of surfacemounted PMSM. The tests show that the proposed method can simultaneously accurately estimate stator resistance and rotor flux linkage performance much better than the existing improved hybrid PSOs.

The structure of this paper is as follows. An estimator model for the identification of stator resistance and rotor flux linkage of a PMSM is described in Section 2. A CLPSO-OBL algorithm is proposed in Section 3 where the optimization procedure and steps are described. Experimental results and the analysis are given in Section 4. Finally, some conclusions and future work are presented in Section 5.

\section{PMSM Model and Design of Parameter Estimation Model}

2.1. PMSM Model. In order to estimate the parameters of a PMSM, the $d q$-axis voltage equations of the machine are used:

$$
\begin{aligned}
& u_{d}=R i_{d}+L_{d} \frac{d i_{d}}{d t}-L_{q} \omega i_{q}, \\
& u_{q}=R i_{q}+L_{q} \frac{d i_{q}}{d t}+L_{d} \omega i_{d}+\psi \omega,
\end{aligned}
$$

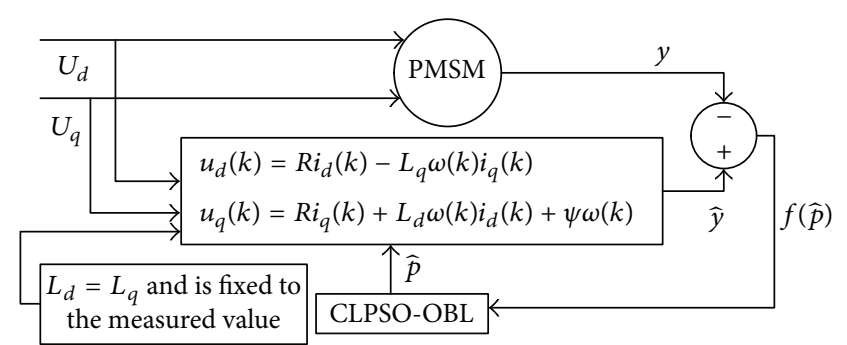

FIGURE 1: PMSM parameter estimation model.

where $\omega, u_{d}, u_{q}, i_{d}$, and $i_{q}$ are electrical angular velocity, $d q$-axis stator voltage, and current and the parameter set $\left\{R, L_{d}, L_{q}, \psi\right\}$ is winding resistance, $d q$-axis inductances, and rotor PM flux linkage, respectively, where $L_{d}=L_{q}=L$ for $S P M S M$. Equation (1) can be discretized as follows when the machine is on steady state:

$$
\begin{aligned}
& u_{d}(k)=R i_{d}(k)-L_{q} \omega(k) i_{q}(k), \\
& u_{q}(k)=R i_{q}(k)+L_{d} \omega(k) i_{d}(k)+\psi \omega(k) .
\end{aligned}
$$

In real application, the $d q$-axis inductance belongs to the slowly varying parameters within a certain range compared to the stator resistance and the rotor PM flux linkage, so it can be considered constant and fixed to measured values during parameter estimation process. The parameters vector $\{R, \psi\}$ is unknown and needs to be identified in this study. The estimation of the parameters is formulated as a system optimization problem by optimizing the designed objective function in this study. The fitness function is defined as a discrepancy between the model output and the measured actual system output. The estimation of the parameters can be addressed as an optimization problem via optimizing objective function. The PMSM parameter estimation model is as shown in Figure 1. From Figure 1, by comparing model output and actual output, the objective function (3) for estimating the winding resistance and rotor flux linkage is designed as follows:

$$
\begin{aligned}
f(\widehat{p}) & =F(R, \psi) \\
& =\frac{1}{n} \sum_{k=1}^{n}\left(\left|u_{d}(k)-\widehat{u}_{d}(k)\right|+\left|u_{q}(k)-\widehat{u}_{q}(k)\right|\right),
\end{aligned}
$$

where the symbol " " means that they are computed voltages by the estimated parameters and measured value. The actual machine parameter values can be obtained if the designed objective function is minimized by the proposed CLPSOOBL. Actually, the objective function (3) is a nonlinear multidimensional function optimization problem and has many local points as it relates to the actual motor system whose system variables are easy to change.

\section{The Proposed Improved CLPSO Using OBL}

3.1. Principle of Basic PSO Algorithm. Inspired by the intelligent behavior of birds, a swarm of particles are to find 
a better solution. Assuming, in a $d$-dimensional solution space, each particle $i$ is composed of the velocity vector $V_{i}=$ $\left\{V_{i 1}, V_{i 2}, \ldots, V_{i d}\right\}$ and position vector $X_{i}=\left\{X_{i 1}, X_{i 2}, \ldots, X_{i d}\right\}$, the velocity and position of $i$ th particle are modified as given in

$$
\begin{aligned}
V_{i d}(t+1)= & \phi V_{i d}+\lambda 1 \\
& * \operatorname{rand}_{1}()\left(\text { Pbest }_{i d}(t)-X_{i d}(t)\right) \\
& +\lambda 2 \\
& * \operatorname{rand}_{2}()\left(\text { Gbest }_{d}(t)-X_{i d}(t)\right), \\
X_{i d}(t+1)= & X_{i d}(t)+V_{i d}(t+1),
\end{aligned}
$$

where Pbest $_{i d}$ represents the best position found by the $i$ th particle up to now and Gbest ${ }_{d}$ is the best particle among the entire population. $\lambda 1$ and $\lambda 2$ are acceleration coefficients, $\phi$ is inertia weight factor, and rand $_{1}$ and $\operatorname{rand}_{2}$ are uniformly distributed numbers generated randomly on $[0,1]$.

3.2. Principle of Basic CLPSO Algorithm. The CLPSO was first introduced by Liang et al. to solve multimodel problems [16]. The searching mechanism of CLPSO is that any particle's velocity vector could be selected to update the velocity of the particle that needs to be modified according to the designed learning probability among the swarm. This modification strategy can effectively avoid diversity losses of convergence for population during the search process. The modifications of the velocity and position of $i$ th particle in CLPSO are as given in

$$
\begin{aligned}
v_{i d}^{k+1} & =w \times v_{i d}^{k}+c \times \operatorname{rand}() \times\left(\text { Pbest }_{f i(d)}-x_{i d}^{k}\right), \\
v_{i d}^{k} & =\min \left(v_{\max }^{k}, \max \left(v_{\min }^{d}, v_{i d}^{k}\right)\right), \\
x_{i d}^{k+1} & =x_{i d}^{k}+v_{i d}^{k+1},
\end{aligned}
$$

where $f i=[f i(1), f i(2), \ldots, f i(d)]$ defined which particle the $i$ th particle should learn from and Pbest ${ }_{f i(d)}$ could be any particle's dimension corresponding value or could be its selfcorresponding value, which is determined by the learning probabilities $p c$, the details as in [16]. The basic ideas are as given: if the random number is greater than $p c_{i}$, this dimension will be learning from the particle's own Pbest; otherwise, it learns from other particles' Pbest. There is one drawback for existing CLPSO algorithm; that is, once the group falls into local optimum, the search behavior of whole swarm will easily get similarity among the total population, as there is no effective mechanism to guarantee the escape from local optima.

3.3. OBL for Pbest's Learning. The Pbest's positions are used as the exemplars to lead the flying direction of the whole population, so the search status of Pbest particles is important for the CLPSO. In order to enhance global convergence performance of the Pbest particles, an OBL strategy is introduced into CLPSO. The OBL was a machine learning method described in detail by Rahnamayan et al. [17], which is a simple technique that allows the population-based algorithms to

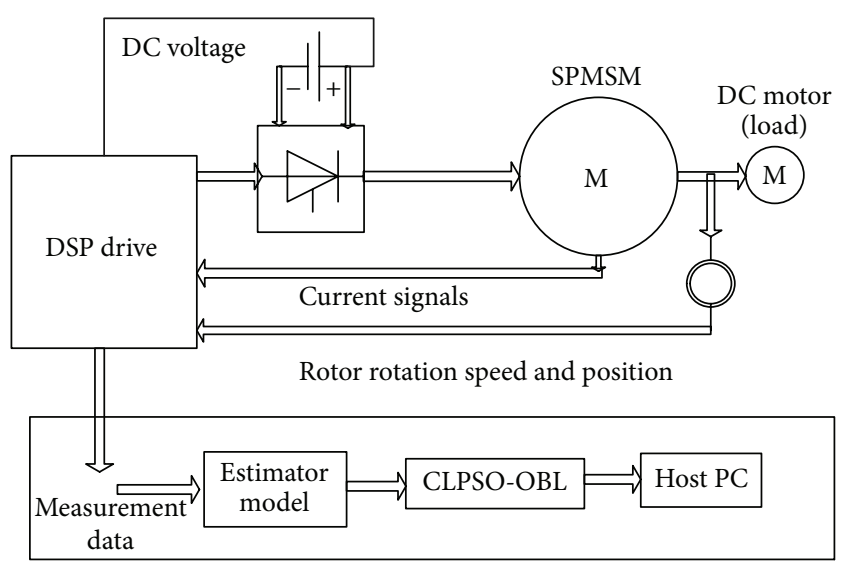

FIGURE 2: The hardware and software platform for the identification of stator resistance and rotor flux linkage in SPMSM.

search for an optimal point in the counter direction and the current search simultaneously. The basic idea is that when a solution is being exploited in a direction, it executes a search in the opposite direction simultaneously as given in

$$
\tilde{x}=a+b-x,
$$

where $x$ is real number in the interval $[a, b]$ and $\tilde{x}$ is the opposite number of $x$. This definition can also be extended to multidimensional space. In $D$-dimensional space, where $x_{1}, x_{2}, \ldots, x_{D} \in R$ and $x_{i} \in\left[a_{i}, b_{i}\right]$, the point $x_{i}$ can be defined as

$$
\tilde{x}_{i}=a_{i}+b_{i}-x_{i}
$$

The OBL machine learning technique is applied into CLPSO and executes opposite learning for Pbest's particles as given in

$$
\begin{aligned}
\text { oPbest }_{i d(j)} & =O a_{d}(t)+O b_{d}(t)-\text { Pbest }_{i d(j)}, \\
O a_{d}(t) & =\min \left(\text { Pbest }_{i d(j)}\right), \\
O b_{d}(t) & =\max \left(\text { Pbest }_{i d(j)}\right) .
\end{aligned}
$$

After the above modification, evaluate the fitness value $(F i t(X i))$ of oPbest $_{n}$ (opposition Pbest ${ }_{i}$ ) and update Pbest $t_{i}$ as $\left(\right.$ Pbest $_{i} \leftarrow$ Pbest $_{i} \cup$ oPbest $\left.{ }_{i}\right)$. The proposed OBL learning operator can help Pbest particles jump out of the local optima and obtain a global convergence performance in the CLPSO.

\section{Experimental Verification}

The proposed estimator is verified by experiments in this section. The Digital Signal Process (DSP) based vector control system and the schematic diagram of testing process are shown in Figure 2. The offline estimation procedure using CLPSO-OBL is as shown in Figure 3. The design parameters of the used prototype machine are detailed as follows: rated speed $(400 \mathrm{rpm})$, rated current $(4 \mathrm{~A}), \mathrm{DC}$ link voltage $(36 \mathrm{v})$, nominal terminal wire resistance $(0.043)$, nominal self-inductance $(2.91 \mathrm{mh})$, nominal mutual inductance $(-0.330 \mathrm{mh})$, nominal $d$-axis inductance $(3.24 \mathrm{mh})$, 


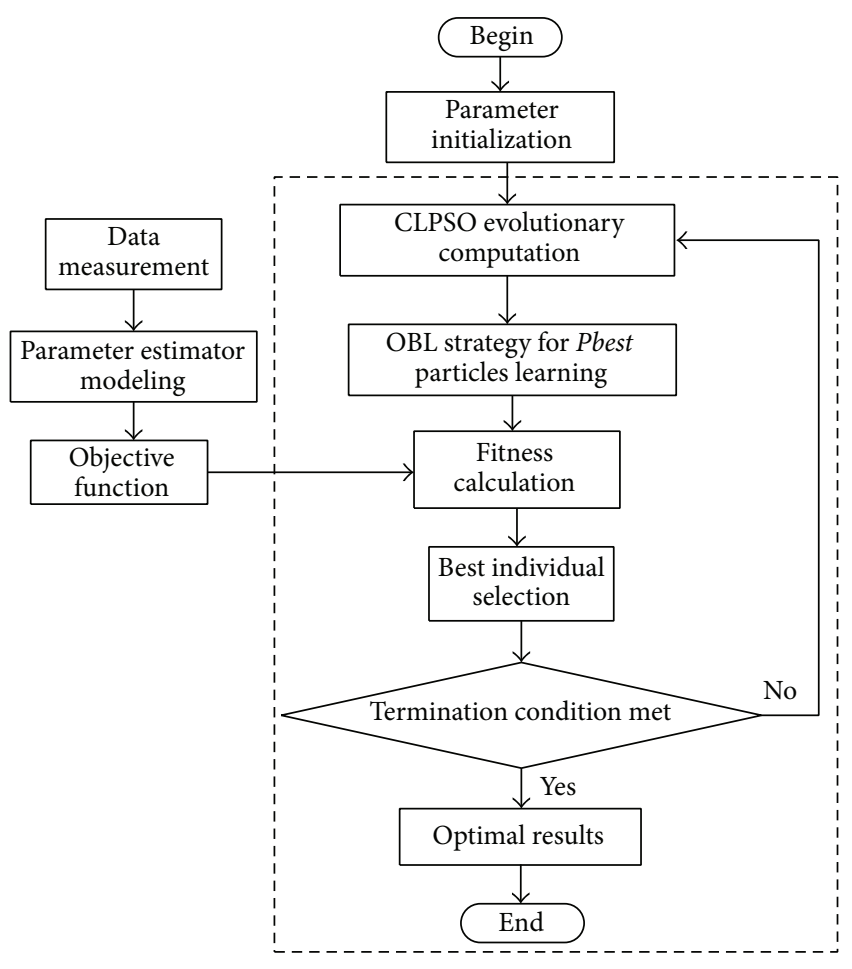

FIgURE 3: The process of parameter estimation in PMSM based on CLPSO-OBL.

nominal $q$-axis inductance $(3.24 \mathrm{mh})$, nominal amplitude of flux induced by magnets $(77.6 \mathrm{mWb})$, number of pole pairs (5), nominal phase resistance $\left(T=25^{\circ} \mathrm{C}\right)(0.330 \Omega)$, and inertia $\left(0.8 e-5 \mathrm{kgm}^{2}\right)$.

$L_{d}=L_{q}=L$ is fixed to one of our prior estimated values [18] (in this research literature $L$ is set to be $3.97(\mathrm{mWb})$ on normal temperature, and $L$ is set to be $3.76(\mathrm{mWb})$ on the heating temperature). As is shown in Figure 3, the estimation of PMSM parameter includes data measurement, parameter estimation modeling, and model parameter optimization procedure. For comparison, the designed PMSM parameter estimation model is also tested by other existing PSOs such as a hybrid PSO with wavelet mutation (HPSOWM) operation method [19], comprehensive learning PSO (CLPSO) method [16], an improved comprehensive learning PSO (A-CLPSO) method [20], and adaptive particle swarm optimization (APSO) method [21]. The basic settings of these PSOs are as follows: the population size is 50 , the maximum generation is 300 , and the number of runs is 30 . All the tested PSOs are using the same measurement data and operated on the same software platform. All experiments are carried out on the same host computer with hardware configuration of Intel ${ }^{\circledR}$ core $^{\mathrm{TM}}-\mathrm{i} 5-2450 \mathrm{M}$ and 4.0 GB DDR3 RAM.

The experiments are carried out under two different work conditions including normal temperature and temperature variation.

Under normal temperature condition, the convergence of different PSOs is shown in Figure 4, the experimental results are depicted in Table 1, and the two parameters' estimated results plotted curve for different PSOs is shown

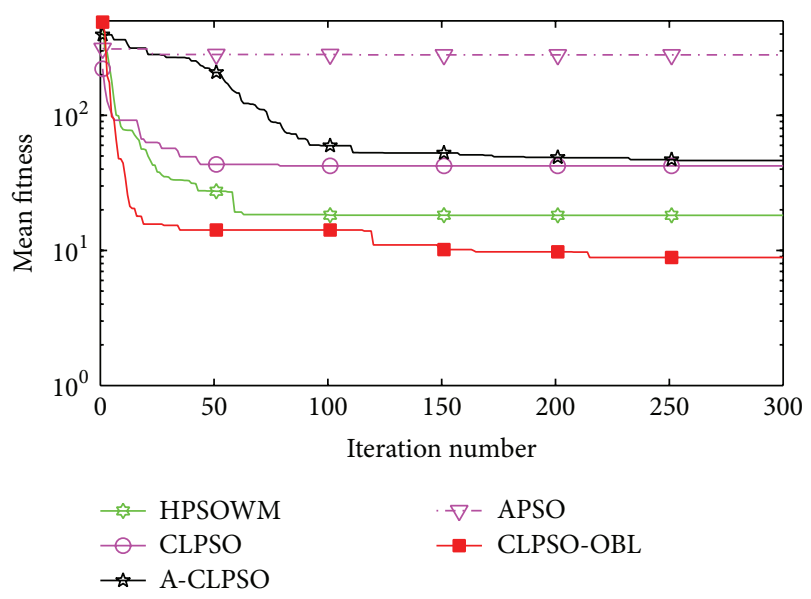

FIgURE 4: The convergence curve of five PSOs on PMSM parameter estimation under normal temperature condition.

TABLE 1: Result of PMSM parameter estimation under normal temperature condition.

\begin{tabular}{lccccc}
\hline $\begin{array}{l}T=300 \\
\text { Parameters }\end{array}$ & HPSOWM & CLPSO & $\begin{array}{c}\text { A-CLPSO } \\
\text { Results }\end{array}$ & APSO & CLPSO-OBL \\
\hline$R(\Omega)$ & 0.339 & 0.329 & 0.333 & 0.346 & 0.334 \\
$\psi(\mathrm{mWb})$ & 0.0790 & 79.29 & 79.20 & 79.21 & 79.16 \\
\hline Fit & & & & & \\
$\quad$ Mean & 18.19 & 42.24 & 46.32 & 280.34 & 8.86 \\
$\quad$ Std. dev. & 24.12 & 31.24 & 29.89 & 212.09 & 2.67 \\
$\quad t$-value & 2.73 & 7.55 & 8.85 & 9.05 & 0 \\
\hline
\end{tabular}

in Figure 5. From Table 1 and Figure 3, it can be seen that the CLPSO-OBL shows the best performances in terms of mean, standard deviations, and $t$-values compared to the existing peer hybrid PSOs (i.e., HPSOWM, CLPSO, A-CLPSO, and APSO). Furthermore, the convergence speed of CLPSO-OBL is faster than other hybrid PSOs as shown in Figure 4.

It is evident that the optimality, convergence, and algorithmic efficiency of CLPSO is improved, thanks to the OBL operator which enhanced the global convergence of CLPSO and pushed it out from the local point. As can be seen from Table 1 , the estimated winding resistance $(0.334 \Omega)$ by the CLPSO-OBL is quite coincident with its nominal value $(0.33 \Omega)$ under normal temperature condition. Also, the estimated flux linkage $\psi(79.16 \mathrm{mWb})$ by CLPSOOBL is quite close to its nominal value $(77.6 \mathrm{mWb})$. The slight difference between the estimated and nominal values of machine parameters may be caused by nonlinearity of machine operation condition.

The parameters of PMSM are easily changed by the environment temperature. In order to check the performance of the proposed method and whether it can track the variation of parameters with the changing temperature condition, a heater is used to heat the prototype PMSM for 20 minutes and then to measure the data for experiment test. The identified results of temperature variation operation condition are listed in Table 2, and the comparison with different PSOs is as 


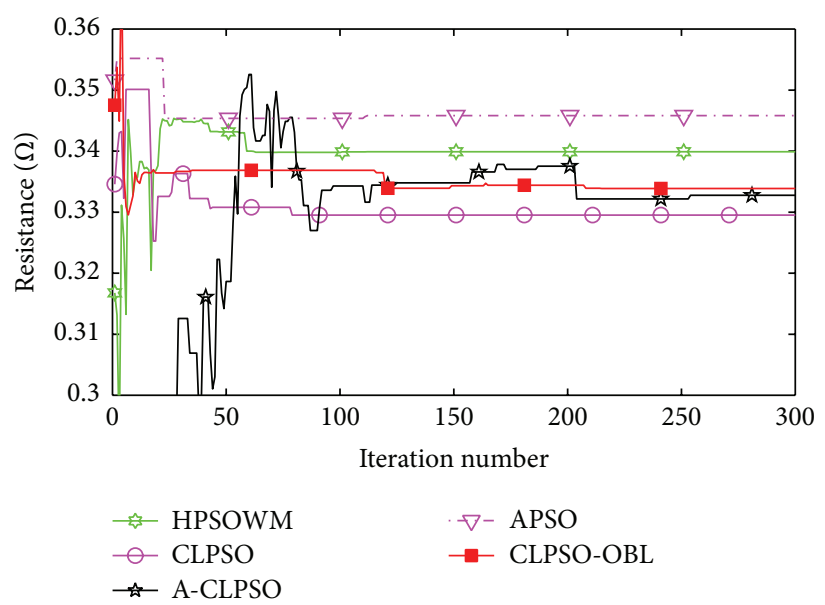

(a) The estimated stator resistance

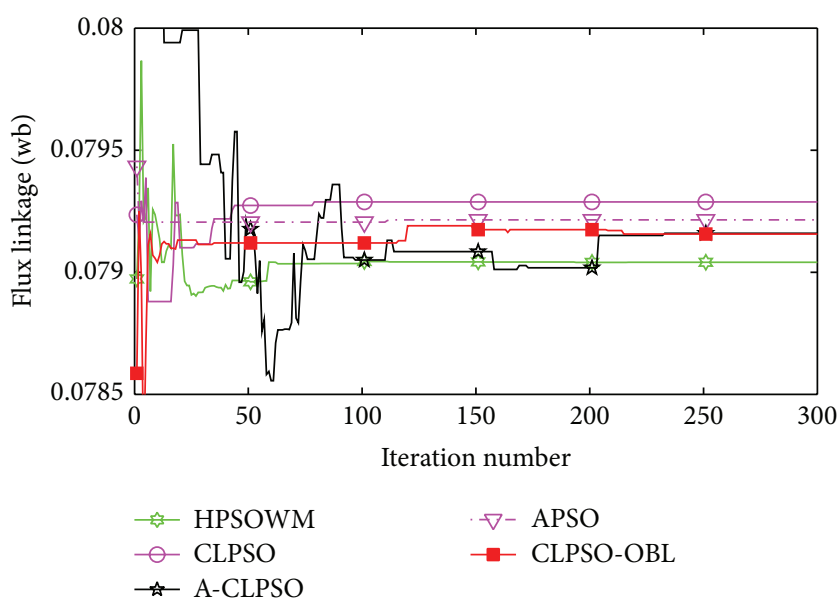

(b) The estimated rotor PM flux linkage

FIGURE 5: The curve of estimated parameters with normal temperature.

TABLE 2: Results of PMSM parameter identification with temperature variation.

\begin{tabular}{lccccc}
\hline $\begin{array}{l}T=300 \\
\text { Parameters }\end{array}$ & HPSOWM & CLPSO & $\begin{array}{c}\text { A-CLPSO } \\
\text { Results }\end{array}$ & APSO & CLPSO-OBL \\
\hline$R(\Omega)$ & 0.455 & 0.463 & 0.455 & 0.475 & 0.454 \\
$\psi(\mathrm{mWb})$ & 0.0769 & 76.69 & 76.92 & 76.4 & 76.9 \\
\hline Fit & & & & & \\
$\quad$ Mean & 9.06 & 48.92 & 17.09 & 265.39 & 8.47 \\
$\quad$ Std. dev. & 2.32 & 29.72 & 9.45 & 126.78 & 0.52 \\
$\quad t$-value & 1.72 & 9.62 & 6.43 & 14.33 & 0 \\
\hline
\end{tabular}

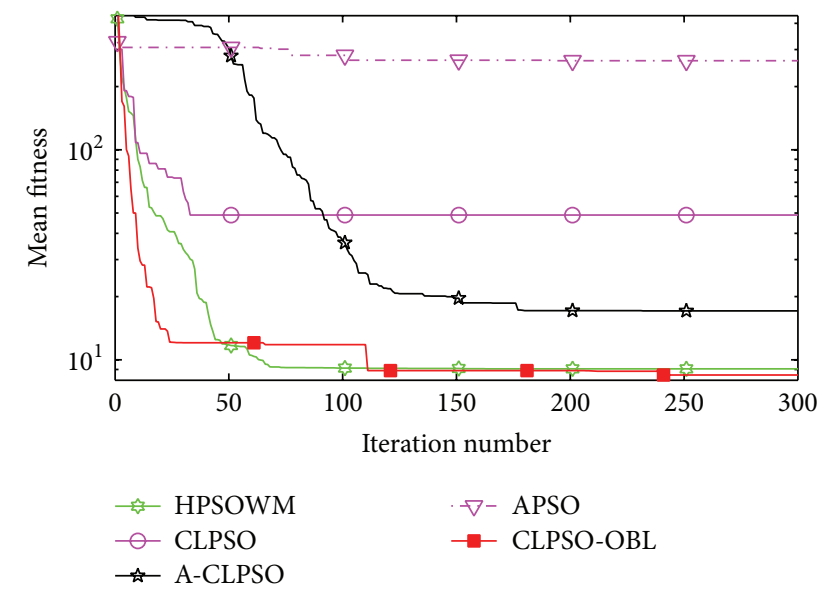

FIgure 6: The convergence curve of several PSOs on PMSM parameter estimation under temperature variation (with heating for $20 \mathrm{~min})$.

shown in Figures 6 and 7, respectively. From Table 2, it is clear that CLPSO-OBL outperforms other peer PSOs in terms of mean, standard deviation, and $t$-test values. From Figure 5, it can be noticed that CLPSO-OBL has a faster convergence speed than other hybrid PSOs.
The analysis results show that the estimated winding resistance $R$ and rotor flux linkage $\psi$ vary with the changing temperature condition. For example, the estimated winding resistance value increases from $0.334(\Omega)$ to $0.454(\Omega)$ with heating for 20 minutes under high temperature. This phenomenon indicates that the metal resistance value increases with increasing temperature due to metal thermal efficiency. The estimated rotor flux linkage decreases from $79.16(\mathrm{mWb})$ to $76.9(\mathrm{mWb})$, the abrupt drop in the estimated rotor flux linkage after 20-minute heating. This phenomenon indicates that magnetic field density decreases with the increasing temperature. These results show that the proposed parameter estimator can simultaneously track the stator resistance and rotor PM flux linkage of PMSM.

\section{Conclusion}

Stator resistance and rotor PM flux linkage are important for controller design and condition monitoring of permanent magnet synchronous machine (PMSM) system. In this study, an improved CLPSO with OBL strategy is proposed for estimating stator resistance and rotor PM flux in surfacemounted PMSM. In the presented algorithm framework, an OBL strategy is used for Pbest particles reinforcement learning to improve the dynamic performance and global optimization ability of the CLPSO. The proposed method not only retains the advantages of diversity in the CLPSO but also has inherited global exploration capability of the OBL. Finally, the proposed method has been successfully applied into the estimation of the stator resistance and rotor PM flux linkage of SPMSM. The experimental results show that the CLPSO-OBL has better performance inestimating winding resistance and rotor PM flux linkage compared to the existing hybrid PSOs. Furthermore, the proposed method can track the variation of machine parameters effectively with the changing work condition. Moreover, the proposed parameter estimation model is simple and with fast convergence and easy digital implementation. Thus, the proposed method 


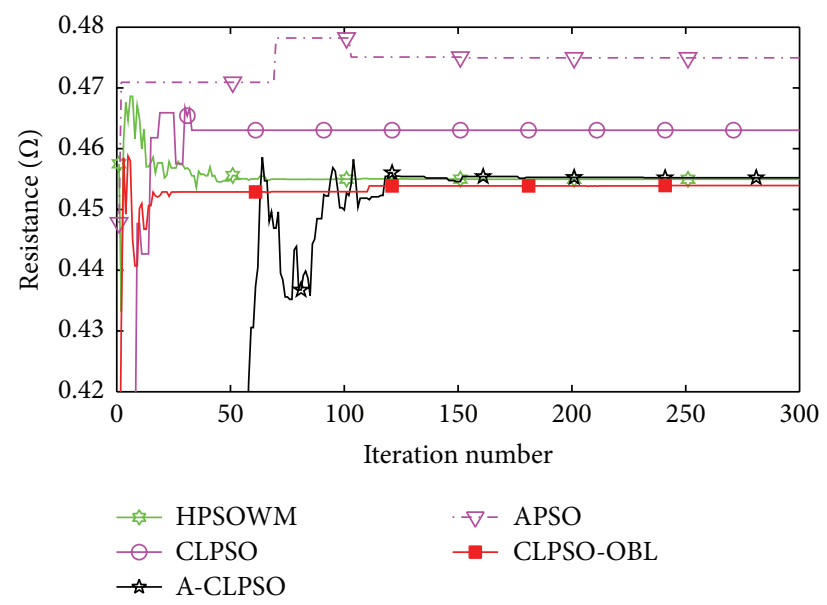

(a) The estimated stator resistance

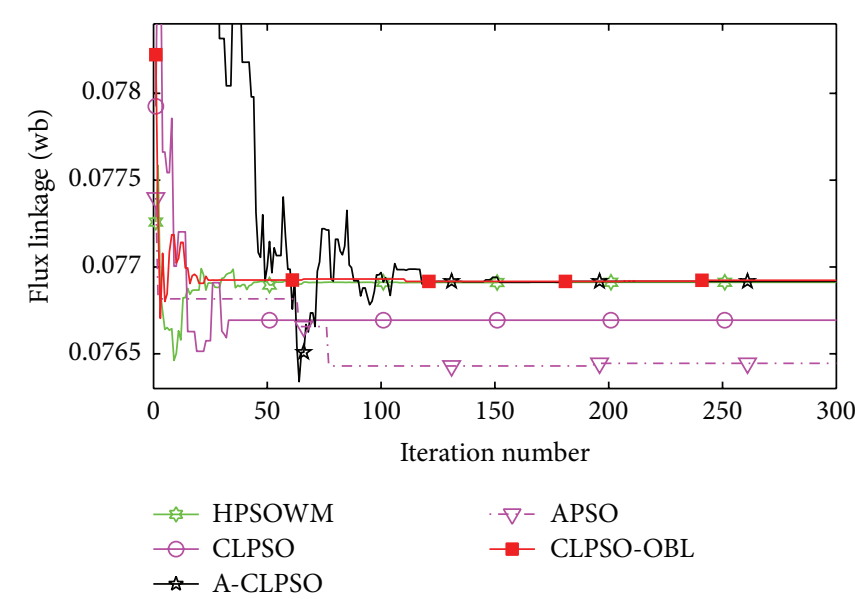

(b) The estimated rotor PM flux linkage

FIGURE 7: Identified parameters under temperature variation (with heating for $20 \mathrm{~min}$ ).

can be used for the condition monitoring of the stator winding and rotor PM flux linkage of PMSM. With the increasing of industrial real-time demand, we will carry it out on Field-Programmable Gate Array (FPGA), and real-time performance control of PMSM will be greatly improved in future.

\section{Competing Interests}

The authors declare that there are no competing interests regarding the publication of this paper.

\section{Acknowledgments}

This work was supported in part by the National Natural Science Foundation of China under Grants 61503134 and 61573299, the China Postdoctoral Science Foundation funded project under Grants 2013M540628 and 2014T70767, and the Hunan Provincial Education Department outstanding youth project under Grant 15B087. The first author (Jian He is an Undergraduate Student) acknowledges the guidance of teacher Xiao-Hua Li who is with the School of Information and Electrical Engineering, Hunan University of Science and Technology.

\section{References}

[1] Z. Chen, J. M. Guerrero, and F. Blaabjerg, "A review of the state of the art of power electronics for wind turbines," IEEE Transactions on Power Electronics, vol. 24, no. 8, pp. 1859-1875, 2009.

[2] Z.-H. Liu, J. Zhang, S.-W. Zhou, X.-H. Li, and K. Liu, "Coevolutionary particle swarm optimization using AIS and its application in multiparameter estimation of PMSM," IEEE Transactions on Cybernetics, vol. 43, no. 6, pp. 1921-1935, 2013.

[3] F. F. M. El-Sousy, "Intelligent optimal recurrent wavelet elman neural network control system for permanent-magnet synchronous motor servo drive," IEEE Transactions on Industrial Informatics, vol. 9, no. 4, pp. 1986-2003, 2013.
[4] S. Kwak, U.-C. Moon, and J.-C. Park, "Predictive-control-based direct power control with an adaptive parameter identification technique for improved AFE performance," IEEE Transactions on Power Electronics, vol. 29, no. 11, pp. 6178-6187, 2014.

[5] Z. H. Liu, X. H. Li, H. Q. Zhang, L. H. Wu, and K. Liu, "An enhanced approach for parameter estimation: using immune dynamic learning PSO based on multi-core architecture," IEEE Systems, Man, and Cybernetics Magazine, vol. 2, no. 1, pp. 26-33, 2016.

[6] Y. C. Shi, K. Sun, L. P. Huang, and Y. Li, "Online identification of permanent magnet flux based on extended Kalman filter for IPMSM drive with position sensorless control," IEEE Transactions on Industrial Electronics, vol. 59, no. 11, pp. 4169-4178, 2012.

[7] E. Monmasson, L. Idkhajine, M. N. Cirstea, I. Bahri, A. Tisan, and M. W. Naouar, "FPGAs in industrial control applications," IEEE Transactions on Industrial Informatics, vol. 7, no. 2, pp. 224-243, 2011.

[8] M. Rashed, P. F. A. MacConnell, A. F. Stronach, and P. Acarnley, "Sensorless indirect-rotor-field-orientation speed control of a permanent-magnet synchronous motor with stator-resistance estimation," IEEE Transactions on Industrial Electronics, vol. 54, no. 3, pp. 1664-1675, 2007.

[9] S. Moreau, R. Kahoul, and J.-P. Louis, "Parameters estimation of permanent magnet synchronous machine without adding extra-signal as input excitation," in Proceedings of the IEEE International Symposium on Industrial Electronics, vol. 1, pp. 371-376, Ajaccio, France, May 2004.

[10] R. Ramakrishnan, R. Islam, M. Islam, and T. Sebastian, "Real time estimation of parameters for controlling and monitoring permanent magnet synchronous motors," in Proceedings of the IEEE International Electric Machines and Drives Conference (IEMDC '09), pp. 1194-1199, Miami, Fla, USA, May 2009.

[11] S. J. Underwood and I. Husain, "Online parameter estimation and adaptive control of permanent-magnet synchronous machines," IEEE Transactions on Industrial Electronics, vol. 57, no. 7, pp. 2435-2443, 2010.

[12] F. F. M. El-Sousy, "Robust wavelet-neural-network slidingmode control system for permanent magnet synchronous motor drive," IET Electric Power Applications, vol. 5, no. 1, pp. 113-132, 2011. 
[13] L. Liu, W. X. Liu, and D. A. Cartes, "Permanent magnet synchronous motor parameter identification using particle swarm optimization," International Journal of Computational Intellig ence Research, vol. 4, no. 2, pp. 211-218, 2008.

[14] Z.-H. Liu, S.-W. Zhou, K. Liu, and J. Zhang, "Permanent magnet synchronous motor multiple parameter identification and temperature monitoring based on binary-modal adaptive wavelet particle swarm optimization," Acta Automatica Sinica, vol. 39, no. 12, pp. 2121-2130, 2013.

[15] Z.-H. Liu, X.-H. Li, L.-H. Wu, S.-W. Zhou, and K. Liu, "GPUaccelerated parallel coevolutionary algorithm for parameters identification and temperature monitoring in permanent magnet synchronous machines," IEEE Transactions on Industrial Informatics, vol. 11, no. 5, pp. 1220-1230, 2015.

[16] J. J. Liang, A. K. Qin, P. N. Suganthan, and S. Baskar, "Comprehensive learning particle swarm optimizer for global optimization of multimodal functions," IEEE Transactions on Evolutionary Computation, vol. 10, no. 3, pp. 281-295, 2006.

[17] R. S. Rahnamayan, H. R. Tizhoosh, and M. M. A. Salama, "Opposition-based differential evolution," IEEE Transactions on Evolutionary Computation, vol. 12, no. 1, pp. 64-79, 2008.

[18] Z.-H. Liu, J. Zhang, X.-H. Li, and Y.-J. Zhang, "Immune coevolution particle swarm optimization for permanent magnet synchronous motor parameter identification," Acta Automatica Sinica, vol. 38, no. 10, pp. 1698-1708, 2012.

[19] S. H. Ling, H. H. C. Iu, K. Y. Chan, H. K. Lam, B. C. W. Yeung, and F. H. Leung, "Hybrid particle swarm optimization with wavelet mutation and its industrial applications," IEEE Transactions on Systems, Man, and Cybernetics, Part B: Cybernetics, vol. 38, no. 3, pp. 743-763, 2008.

[20] H. Wu, J. Geng, R. Jin et al., "An improved comprehensive learning particle swarm optimization and its application to the semiautomatic design of antennas," IEEE Transactions on Antennas and Propagation, vol. 57, no. 10, pp. 3018-3028, 2009.

[21] Z.-H. Zhan, J. Zhang, Y. Li, and H. S.-H. Chung, "Adaptive particle swarm optimization," IEEE Transactions on Systems, Man, and Cybernetics, Part B: Cybernetics, vol. 39, no. 6, pp. 1362-1381, 2009. 


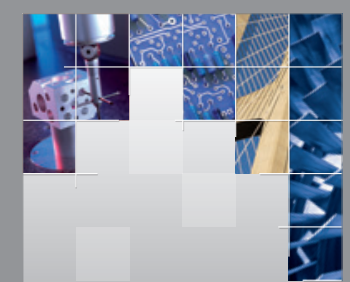

\section{Enfincering}
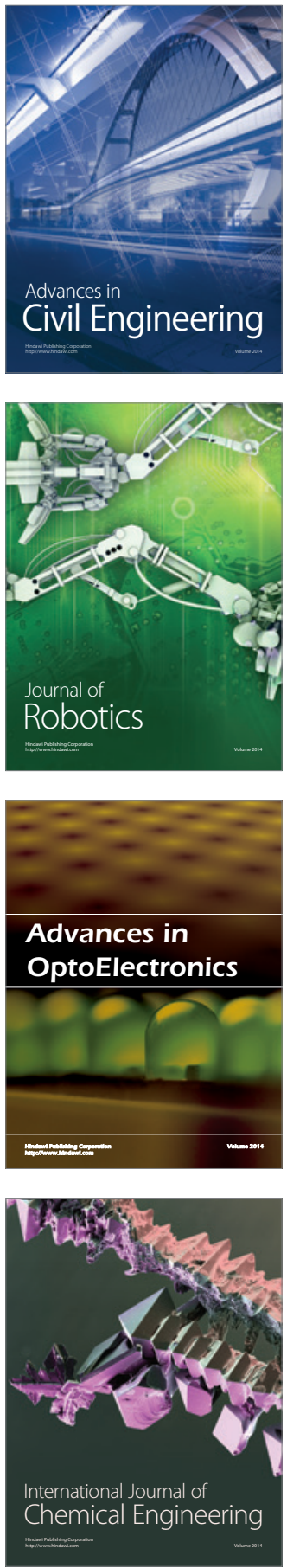

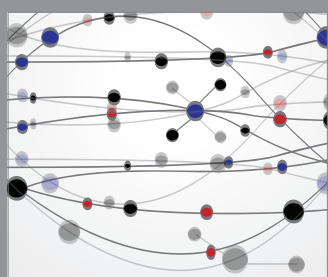

The Scientific World Journal

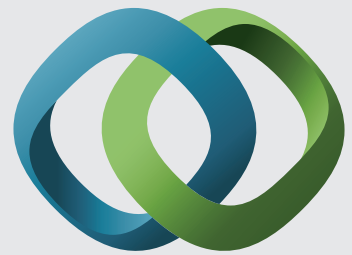

\section{Hindawi}

Submit your manuscripts at

http://www.hindawi.com
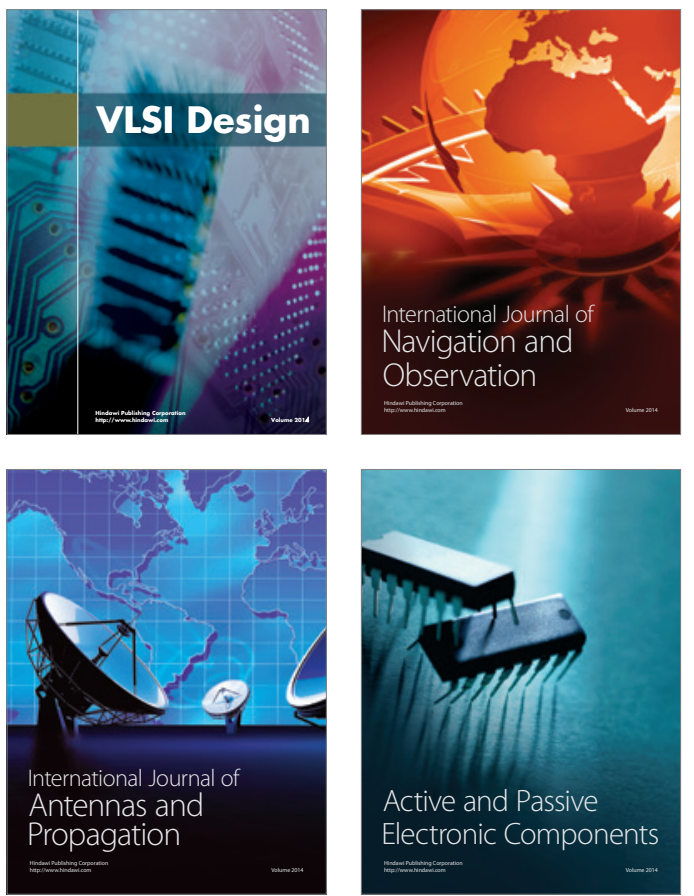
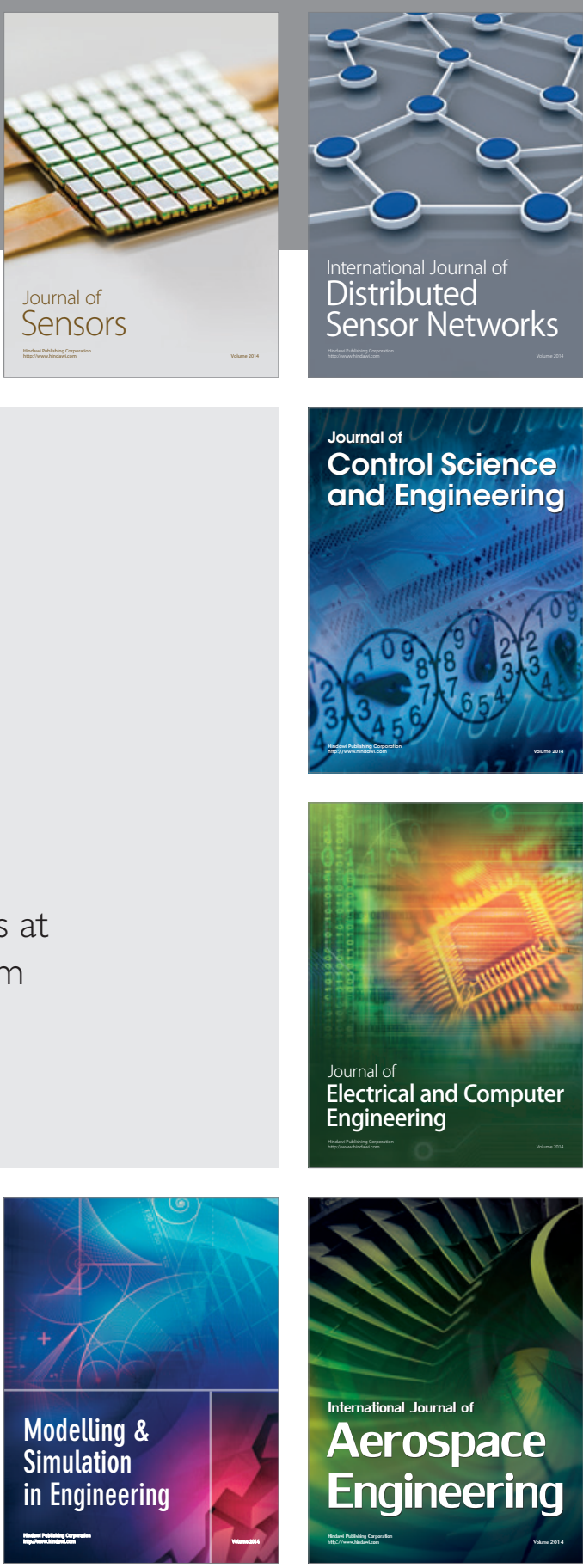

International Journal of

Distributed

Sensor Networks

Journal of

Control Science

and Engineering
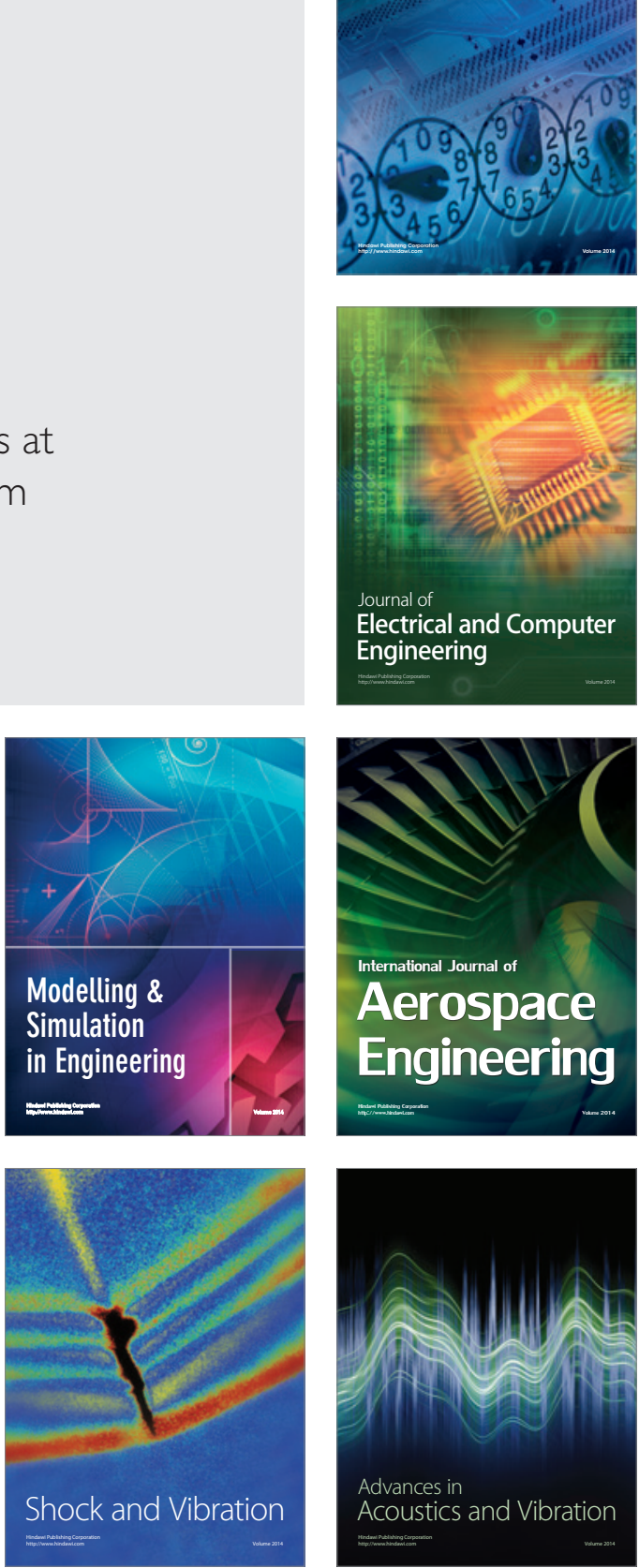